Research Paper

\title{
Neoadjuvant Chemotherapy with Fluorouracil plus Nedaplatin or Cisplatin for Locally Advanced Nasopharyngeal Carcinoma: a Retrospective Study
}

\author{
Tongxin Liu $1,2,3,{ }^{*}$, Quanquan Sun ${ }^{1,2,}{ }^{*}$, Jing Chen ${ }^{1,2,3}$, Bin Li1 1,2,3, Weifeng Qin ${ }^{1,2,3}$, Fangzheng Wang1,2,3, Zhimin \\ $\mathrm{Ye}^{1,2,3}$, Fujun $\mathrm{Hu}^{1,2,3, \mathbb{}}$ \\ 1. Department of Radiation Oncology, Zhejiang Cancer Hospital, Hangzhou, Zhejiang 310022, China \\ 2. Key Laboratory of Radiation Oncology in Zhejiang Province, Hangzhou, Zhejiang 310022, China \\ 3. Key Laboratory of Head \& Neck Cancer Translational Research of Zhejiang Province, Hangzhou, Zhejiang 310022, China \\ * These authors contributed equally to this work \\ $\square$ Corresponding author: FuJun Hu, Department of Radiation Oncology, Zhejiang Cancer Hospital, Hangzhou, Zhejiang 310022, China; E-mail: \\ hufj381@hotmail.com \\ (C) Ivyspring International Publisher. This is an open access article distributed under the terms of the Creative Commons Attribution (CC BY-NC) license \\ (https://creativecommons.org/licenses/by-nc/4.0/). See http://ivyspring.com/terms for full terms and conditions.
}

Received: 2018.05.10; Accepted: 2018.08.15; Published: 2018.09.08

\begin{abstract}
The present study aimed to evaluate the efficacy, toxicity and long-term outcome of nedaplatin or cisplatin combined with 5-fluorouracil neoadjuvant chemotherapy (NF or PF regimen) followed by concurrent chemoradiotherapy (CCRT) for treatment of locally advanced nasopharyngeal carcinoma (NPC). In this study, a total of 186 patients with locally advanced NPC between January 2009 and November 2011 in our center were retrospectively analyzed. 103 cases were received NF neoadjuvant chemotherapy followed by nedaplatin concurrent intensity-modulated radiotherapy (IMRT), and 83 cases were received PF neoadjuvant chemotherapy followed by cisplatin concurrent IMRT. Overall survival (OS), progression-free survival (PFS), local relapse-free survival (LRFS), regional relapse-free survival (RRFS) and distant metastasis-free survival (DMFS), as well as acute toxicities were monitored. Results showed that there were no significant differences in 5-year OS, PFS, LRFS, RRFS and DMFS between NF and PF groups. NF group had a higher incidence of grade $3-4$ neutropenia $(46.6 \%$ vs. $31.3 \%, P=0.035)$ and thrombocytopenia $(17.5 \%$ vs. $7.3 \%, P=0.042)$ compared with $P F$ group. However, NF group was less common to suffer from grade $3-4$ nausea $(1.9 \%$ vs. $24.1 \%, P<0.001)$, vomiting ( $0 \%$ vs. $13.3 \%, P<0.001)$ and weight loss $(0 \%$ vs. $4.8 \%, P=0.025)$. In multivariate analysis, $N$ stage was an independent factor for OS, PFS, RRFS and DMFS. In conclusion, neoadjuvant chemotherapy with fluorouracil plus nedaplatin followed by nedaplatin concurrent with IMRT exhibited similar efficacy but more tolerable toxicity than cisplatin setting, which might be an effective and safe choice for treatment of locally advanced NPC.
\end{abstract}

Key words: nasopharyngeal carcinoma; nedaplatin; neoadjuvant chemotherapy; concurrent chemoradiotherapy

\section{Introduction}

Nasopharyngeal carcinoma (NPC) is a type of malignant tumor of the head and neck, which is prevalent in South China and Southeast Asia [1], with an annual incidence of approximately 20 to 30 per 100,000 [2]. Due to its specific anatomical location and sensitivity to radiation, radiotherapy is the mainstay treatment modality for NPC [3]. In recent years, the revolution from two-dimensional conventional radiotherapy (2DCRT) to intensity-modulated radiotherapy (IMRT) has improved the outcome of
NPC [4]. For early-stage of NPC (T1N0M0), radiotherapy alone is the mainstay treatment modality. However, approximately $70 \%$ of newly diagnosed NPC patients present with locoregionally advanced disease, which are more likely to develop local recurrence and distant metastasis when treated with radiotherapy alone [5]. Several clinical trials have verified the cisplatin-based concurrent chemoradiotherapy (CCRT) as the standard treatment for locally advanced NPC $[6,7]$. However, CCRT is 
insufficient for patients with high-risk factors, such as bulky tumors and/or extensive nodal disease, which has higher potential for distant metastasis [8].

In order to improve the outcome of locally advanced NPC patients, several studies were carried out to evaluate the efficacy of neoadjuvant chemotherapy (NACT) or adjuvant chemotherapy (ACT) in combination with CCRT [9-11]. Hui et al [9] indicated that NACT plus CCRT has improved 3-year overall survival (OS) than CCRT alone in stage III to IVB NPC patients (94\% VS $67.7 \%, P=0.012$ ). Chen et al [12] also found the similar results. Unfortunately, the conflicting findings of the studies by other researchers made the benefit undetermined [10, 13]. Consequently, the role of NACT is still uncertain and need to be confirmed by more research.

Although the regimen of 5-fluorouracil (5-FU) plus cisplatin (CDDP) has been widely used as the standard treatment for NPC, CDDP is well-known for its side effects such as kidney dysfunction and digestive disorders. In order to relieve these side effects caused by CDDP, a second-generation cisplatin analog, nedaplatin (NDP) is developed [14]. Compared with CDDP, NDP has a different molecular structure but similar anticancer potencies, which is associated with a lower incidence of severe digestive symptoms and renal toxicity. Furthermore, numerous studies have indicated that NDP has wildly used in many kinds of tumors for its efficacy and safety, such as esophageal cancer [15], non-small cell lung cancer [16], uterine cervix cancer [17], and some head and neck squamous cell carcinoma $[18,19]$. To date, the research of NDP versus CDDP in locally advanced NPC is mainly on the short-term efficacy and toxicity, but lack of the long-term survival outcome [20]. Recently, Tang et al [21] performed an open-label, non-inferiority, randomised phase 3 trial to confirmed that nedaplatin-based concurrent chemoradiotherapy represents an alternative doublet treatment strategy to cisplatin-based concurrent chemoradiotherapy for patients with locoregional, advanced nasopharyngeal carcinoma. However, they did not explore the potential use of nedaplatin-based chemotherapy as neoadjuvant chemotherapy. Therefore, we performed this retrospective study to determine the efficacy, toxicity and long-term outcome of neoadjuvant chemotherapy with fluorouracil plus NDP or CDDP combined with concurrent chemoradiotherapy in patients with locally advanced nasopharyngeal carcinoma.

\section{Materials and Methods}

\section{Patients}

Between January 2009 and November 2011, 195 pathologically confirmed locally advanced NPC in
Zhejiang Cancer Hospital who received neoadjuvant chemotherapy with fluorouracil plus NDP or CDDP combined with concurrent chemoradiotherapy were retrospectively reviewed. Inclusion criteria was as follows: Pathologically confirmed NPC with Stage III IVB according to the AJCC 2010 staging system[22], there is no evidence of distant metastasis, no uncontrolled medical or psychiatric disease, while the patients were free from any previous malignancy or other concomitant malignant diseases, and Karnofsky Score $\geq 70$. Prior to treatment, all patients had undergone a complete history and physical examination, complete blood cell count, comprehensive serum chemistry profile, electrocardiogram (ECG), fiber-optic nasopharyngoscopy and biopsy, chest X-ray or computed tomography(CT), ultrasonography of the abdomen or computed tomography $(\mathrm{CT})$, bone scan and magnetic resonance imaging (MRI) of the head and neck region. Among the 195 patients, 9 patients were excluded due to limited follow-up information. As a result, a total of 186 patients were left for analysis. Patient and disease characteristics are listed in Table 1. This study was approved by the Research Ethics Committee of Zhejiang Cancer Hospital.

\section{Radiotherapy}

All patients were treated with IMRT using a $6 \mathrm{MV}$ X-ray. Briefly, gross tumor volume of nasopharynx (GTVnx) included the primary tumor and positive retropharyngeal lymph nodes were determined based on clinical and imaging examinations. Metastatic cervical lymph nodes were defined as gross tumor volume of involved cervical lymph nodes (GTVnd). The high-risk region was delineated as clinical target volume (CTV1), which included the GTVnx and GTVnd with a margin of $5-10 \mathrm{~mm}$, entire nasopharynx, inferior two-thirds of the sphenoid sinus, the anterior third of the clivus, pterygoid fossae, posterior third of nasal cavity and maxillary sinuses, retropharyngeal nodes, parapharyngeal space, and the drainage of the upper neck. Low risk region was defined as CTV2, which included CTV1 plus a margin of 3-5 $\mathrm{mm}$, the lower neck and the supraclavicular lymphatic drainage region. The planning target volume (PTV) was defined as the area from $3-5 \mathrm{~mm}$ outside the CTV or GTV. The dose prescribed was as follows: 66-70 Gy to the GTVnx PTV and 66-70 Gy to the GTVnd PTV, 60 Gy to the CTV1 PTV, 54 Gy to the CTV2 PTV. The total dose of the GTVnx PTV, GTVnd PTV, CTV1 PTV and CTV2 PTV was given in 30-33 fractions. Furthermore, according to the Radiation Therapy Oncology Group 0225 protocol [23], maximum dose of each organ at risk (OAR) was below its tolerance limit. 
All patients received one fraction daily for five days per week. Pinnacle version 7.6 planning system was used to design all the plans.

\section{Chemotherapy}

In the PF group, neoadjuvant chemotherapy regimen comprised $75 \mathrm{mg} / \mathrm{m}^{2}$ of CDDP on day 1 and $800 \mathrm{mg} / \mathrm{m}^{2} / \mathrm{d}$ of 5 -fluorouracil for continuous intravenous infusion 120 hours in a cycle of 21 days for 2 to 3 cycles, followed by IMRT concurrent with CDDP at a dose of $80 \mathrm{mg} / \mathrm{m}^{2}$ altogether for 3 days in a cycle of 21 days for 2 to 3 cycles or weekly intravenous CDDP at $40 \mathrm{mg} / \mathrm{m}^{2}$ for up to 6 cycles. In the NF group, the neoadjuvant chemotherapy regimen comprised $75 \mathrm{mg} / \mathrm{m}^{2}$ of NDP on day 1 and $800 \mathrm{mg} / \mathrm{m}^{2} / \mathrm{d}$ of 5-fluorouracil for continuous intravenous infusion 120 hours in a cycle of 21 days for 2 to 3 cycles, followed by IMRT concurrent with NDP at a dose of $80 \mathrm{mg} / \mathrm{m}^{2}$ every 3 weeks for 2 to 3 cycles or $40 \mathrm{mg} / \mathrm{m}^{2} \mathrm{NDP}$ weekly for up to 6 cycles.

\section{Follow-up and treatment assessment}

Treatment induced toxicities were scored according to the National Cancer Institute Common Terminology Criteria for Adverse Events version 3.0. All patients were evaluated weekly during the treatment, and regularly followed up in our hospital after completion of the treatment once every 3 months in the first 2 years, once every 6 months from the third to the fifth years, and then once every year thereafter until death or their last follow-up appointment. The endpoint of the final follow-up was December 2017, and the median follow-up period was 68 months (ranging from 16 to 97 months). The patients who failed to meet follow-up requirements more than twice were excluded. Actually, our follow-up assessments consist of patient history, physical examination, fiber-optic nasopharyngoscopy and biopsy, chest X-ray or CT, ultrasonography of the abdomen or CT, and MRI examination for the head and neck. Furthermore, when the patients showed the symptoms of bone pain, whole-body bone scan would be performed.

\section{Statistical Analysis}

Statistical analyses were performed using SPSS v18.0 software (SPSS, Chicago, IL, USA). Characteristics of patients were compared via chi-square test or Fisher's exact test. The Kaplan-Meier method was used to calculate the overall survival (OS) rate, local relapse-free survival (LRFS) rate, regional relapse-free survival (RRFS) rate, distant metastasis-free survival (DMFS) rate and progression-free survival (PFS) rate. Multivariate analysis was estimated using the Cox proportional hazards model. Two-sided $P$ values $<0.05$ were considered to be statistically significant.

\section{Results}

\section{Patient Characteristics}

The patients and disease characteristics of 186 cases are listed in Table 1. Among the 186 patients, 103 patients were included in the NF group and 83 patients were included in the PF group. According to the AJCC 2010 staging system, patients in our research were divided into stage III (123 patients; $66.1 \%$ ), stage IVA (34 patients; $18.3 \%$ ) and stage IVB (29 patients; $15.6 \%$ ). Between the two groups, there were no statistically significant differences of the proportional distribution of age ( $\leq 50$ vs $>50)$, sex, histology, T stage, $\mathrm{N}$ stage, AJCC clinical stage and cycle of neoadjuvant chemotherapy (all $\mathrm{P}$ value $>0.05$ ).

Table 1. Patient and disease characteristics

\begin{tabular}{|c|c|c|c|}
\hline Characteristic & NF $(n=103)$ & $\mathrm{PF}(\mathrm{n}=83)$ & $P$ value \\
\hline Age (years) & & & 0.933 \\
\hline$\leq 50$ & $54(52.4 \%)$ & $43(51.8 \%)$ & \\
\hline$>50$ & $49(47.6 \%)$ & $40(48.2 \%)$ & \\
\hline Gender & & & 0.783 \\
\hline Male & $65(63.1 \%)$ & $54(65.1 \%)$ & \\
\hline Female & $38(36.9 \%)$ & $29(34.9 \%)$ & \\
\hline Histology & & & 0.931 \\
\hline WHO I & $17(16.5)$ & $13(15.7)$ & \\
\hline WHO II & 14 (13.6) & $10(12.0)$ & \\
\hline WHO III & $72(69.9)$ & $60(72.3)$ & \\
\hline T stage & & & 0.542 \\
\hline $\mathrm{T} 1$ & $7(6.8 \%)$ & $3(3.6 \%)$ & \\
\hline $\mathrm{T} 2$ & $14(13.6 \%)$ & $8(9.6 \%)$ & \\
\hline T3 & $59(57.3 \%)$ & $55(66.3 \%)$ & \\
\hline $\mathrm{T} 4$ & $23(22.3 \%)$ & $17(20.5 \%)$ & \\
\hline N stage & & & 0.172 \\
\hline No & $14(13.6 \%)$ & $5(6.0 \%)$ & \\
\hline N1 & $20(19.4 \%)$ & $19(22.9 \%)$ & \\
\hline N2 & $50(48.5 \%)$ & $49(59.0 \%)$ & \\
\hline N3 & $19(18.4 \%)$ & $10(12.0 \%)$ & \\
\hline AJCC stage & & & 0.488 \\
\hline III & $66(64.1 \%)$ & $57(68.7 \%)$ & \\
\hline IVA & $18(17.5 \%)$ & $16(19.3 \%)$ & \\
\hline IVB & $19(18.4 \%)$ & $10(12.0 \%)$ & \\
\hline NAC cycle & & & 0.702 \\
\hline 1 & $17(16.5)$ & $12(14.5)$ & \\
\hline $2-3$ & $86(83.5)$ & $71(85.5)$ & \\
\hline
\end{tabular}

$\mathrm{NF}$, nedaplatin combined with 5-fluorouracil neoadjuvant chemotherapy; PF, cisplatin combined with 5-fluorouracil neoadjuvant chemotherapy; NAC neoadjuvant chemotherapy.

\section{Efficacy}

The tumor response three months after completion of therapy in the two groups were depicted in Table 2. Responses were classified as complete response (CR), partial response (PR), stable disease (SD) or progressive disease (PD), according to the RECIST criteria. As for the response of primary tumor, 97 cases $(94.2 \%)$ of CR and 6 cases $(5.8 \%)$ of PR in the NF group, while 80 cases $(96.4 \%)$ of CR and 3 cases $(3.6 \%)$ of PR in the PF group. As for the response 
of nodes, 90 cases $(87.4 \%)$ of CR and 13 cases $(12.6 \%)$ of PR in the NF group, versus 70 cases $(84.3 \%)$ of CR and 13 cases $(15.7 \%)$ of PR in the PF group. There was no significant difference in $C R$ rate either in the response of primary tumor or the response of nodes between the two groups (all $P$ value $>0.05$ ).

Table 2. Tumor response three months after chemoradiotherapy in PF or NF group

\begin{tabular}{|c|c|c|c|c|c|c|c|c|}
\hline & \multicolumn{2}{|c|}{$\begin{array}{l}\text { Response of primary } \\
\text { tumor }(\%)\end{array}$} & \multirow[t]{2}{*}{$X^{2}$} & \multirow[t]{2}{*}{$P$} & \multicolumn{2}{|c|}{ Response of nodes (\%) } & \multirow[t]{2}{*}{$X^{2}$} & \multirow[t]{2}{*}{$P$} \\
\hline & CR & PR & & & CR & PR & & \\
\hline $\mathrm{NF}$ & $97(94.2)$ & $6(5.8)$ & 0.488 & 0.485 & $90(87.4)$ & 13 (12.6) & 0.354 & 0.552 \\
\hline PF & $80(96.4)$ & $3(3.6)$ & & & $70(84.3)$ & 13 (15.7) & & \\
\hline
\end{tabular}

\section{Acute Toxicity}

Acute toxicities were assessed between the two groups and are summarized in Table 3 . In the NF group, 67 patients $(65 \%)$ had grade $0-2$ leucopenia, 36 (35\%) had grade $3-4$. While 53 patients $(63.9 \%)$ in the PF group had grade 0-2 leucopenia, and 30 (36.1\%) had grade 3-4. There were no significant differences between the two groups $(P=0.866)$. However, more patients in the NF group suffered from grade 3-4 neutropenia and grade 3-4 thrombocytopenia than those in the PF group (46.6\% vs $31.3 \%, P=0.035$ and $17.5 \%$ vs $7.3 \%, P=0.042$, respectively). While it was common to suffer from nausea, vomiting and weight loss for patients in the PF groups with $\mathrm{P}$ values of $<0.001$ and 0.025 , respectively. There were no significantly differences between the two groups in anemia, dermatitis, stomatitis (mucositis), xerostomia,

A

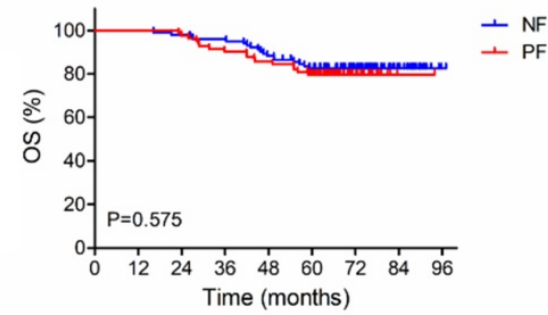

liver or kidney dysfunction, hypokalemia or hyponatremia, with all $\mathrm{P}$ value $>0.05$.

Table 3. Frequency of acute toxicities from the two groups by type and grade

\begin{tabular}{|c|c|c|c|c|c|c|}
\hline \multirow[t]{2}{*}{ Acute Toxicities } & \multicolumn{2}{|l|}{ NF } & \multicolumn{2}{|l|}{$\mathrm{PF}$} & \multirow[t]{2}{*}{ Z } & \multirow[t]{2}{*}{$P$} \\
\hline & $\begin{array}{l}\text { Grades } 0-2 \\
\mathrm{n}(\%)\end{array}$ & $\begin{array}{l}\text { Grades } \\
3-4 \mathrm{n}(\%)\end{array}$ & $\begin{array}{l}\text { Grades } \\
0-2 \mathrm{n}(\%)\end{array}$ & $\begin{array}{l}\text { Grades } \\
3-4 \mathrm{n}(\%)\end{array}$ & & \\
\hline \multicolumn{7}{|l|}{ Hematologic } \\
\hline Leucopenia & $67(65.0)$ & $36(35.0)$ & $53(63.9)$ & $30(36.1)$ & 0.169 & 0.866 \\
\hline Neutropenia & $55(53.4)$ & 48 (46.6) & $57(68.7)$ & $26(31.3)$ & 2.110 & 0.035 \\
\hline Thrombocytopenia & $85(82.5)$ & $18(17.5)$ & $76(92.7)$ & $6(7.3)$ & 2.037 & 0.042 \\
\hline Anemia & $103(100.0)$ & $0(0.0)$ & $83(100.0)$ & $0(0.0)$ & 0.000 & 1.000 \\
\hline \multicolumn{7}{|l|}{ Non-Hematologic } \\
\hline Vomiting & $103(100.0)$ & $0(0.0)$ & $72(86.7)$ & $11(13.3)$ & 3.799 & $<0.001$ \\
\hline Nausea & $101(98.1)$ & $2(1.9)$ & $63(75.9)$ & $20(24.1)$ & 4.638 & $<0.001$ \\
\hline Weight loss & $103(100.0)$ & $0(0.0)$ & $79(95.2)$ & $4(4.8)$ & 2.246 & 0.025 \\
\hline Dermatitis & $95(92.2)$ & $8(7.8)$ & $80(96.4)$ & $3(3.6)$ & 1.190 & 0.234 \\
\hline $\begin{array}{l}\text { Stomatitis } \\
\text { (mucositis) }\end{array}$ & $90(87.4)$ & 13 (12.6) & $74(89.2)$ & $9(10.8)$ & 0.372 & 0.710 \\
\hline Xerostomia & $101(98.1)$ & $2(1.9)$ & $83(100.0)$ & $0(0.0)$ & 1.273 & 0.203 \\
\hline Liver dysfunction & $98(95.1)$ & $5(4.9)$ & $77(92.8)$ & $6(7.2)$ & 0.681 & 0.496 \\
\hline $\begin{array}{l}\text { Kidney } \\
\text { dysfunction }\end{array}$ & $103(100.0)$ & $0(0.0)$ & $83(100.0)$ & $0(0.0)$ & 0.000 & 1.000 \\
\hline Hypokalemia & $103(100.0)$ & $0(0.0)$ & $81(97.6)$ & $2(2.4)$ & 1.580 & 0.114 \\
\hline Hyponatremia & $103(100.0)$ & $0(0.0)$ & 82 (98.8) & $1(1.2)$ & 1.114 & 0.265 \\
\hline
\end{tabular}

\section{Survival outcomes}

For the 186 patients in our study, the median follow-up period was 68 months (ranging from 16 to 97 months). As shown in Figure 1 and Table 4, 5-year OS, PFS, LRFS, RRFS and DMFS rates did not differ significantly between NF and PF groups (OS: $82.4 \%$ vs $79.4 \%, P=0.575$; PFS: $72.6 \%$ vs $68.7 \%, P=0.469$; LRFS: $86.2 \%$ vs $92.6 \%, P=0.211$; RRFS: $91.0 \%$ vs $87.7 \%$, $P=0.485$; DMFS: $80.7 \%$ vs $77.0 \%, P=0.369$ ).

B

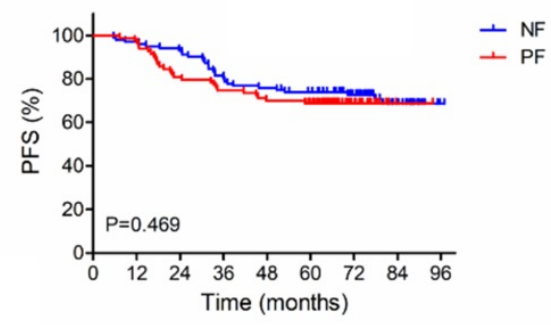

C

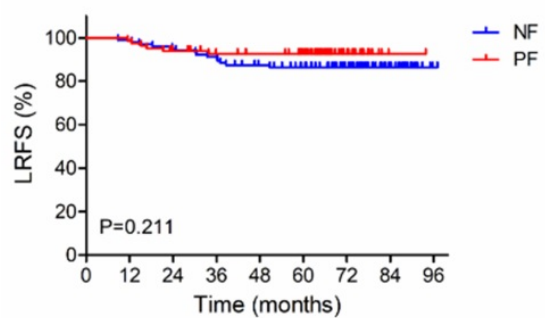

D

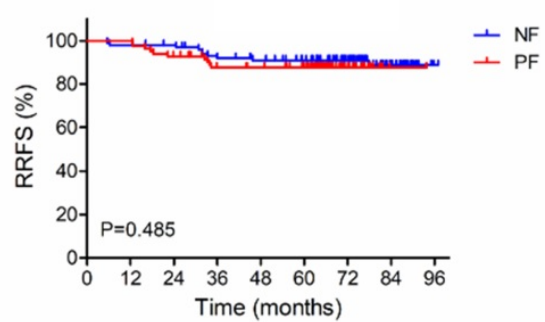

E

Figure 1. Kaplan-Meier survival curves for neoadjuvant chemotherapy with fluorouracil plus nedaplatin or cisplatin followed by concurrent chemoradiotherapy in the 186 patients with stage III-IVb nasopharyngeal carcinoma. (A) Overall survival. (B) Progression-free survival. (C) Local relapse-free survival. (D) Regional relapse-free survival. (E) Distant metastasis-free survival. P-values were calculated using the log-rank test. 
Table 4. Effect of prognostic factors on survival in univariate analysis

\begin{tabular}{|c|c|c|c|c|c|c|c|c|c|c|}
\hline \multirow[t]{2}{*}{ Factors } & \multicolumn{2}{|c|}{$5 y-O S$} & \multicolumn{2}{|c|}{$5 y$-PFS } & \multicolumn{2}{|c|}{ 5y-LRFS } & \multicolumn{2}{|c|}{ 5y-RRFS (\%) } & \multicolumn{2}{|c|}{$5 y-D M F S$} \\
\hline & $\%$ & $P$ & $\%$ & $P$ & $\%$ & $P$ & $\%$ & $P$ & $\%$ & $P$ \\
\hline Gender & & 0.635 & & 0.186 & & 0.945 & & 0.270 & & 0.210 \\
\hline Male & 79.6 & & 67.7 & & 88.8 & & 88.1 & & 75.9 & \\
\hline Female & 83.6 & & 76.1 & & 89.2 & & 92.0 & & 84.2 & \\
\hline Age (years) & & 0.939 & & 0.678 & & 0.515 & & 0.473 & & 0.938 \\
\hline$\leq 50$ & 81.3 & & 71.9 & & 90.5 & & 88.3 & & 78.7 & \\
\hline$>50$ & 80.8 & & 69.6 & & 87.3 & & 90.8 & & 79.2 & \\
\hline Histology & & 0.945 & & 0.782 & & 0.148 & & 0.885 & & 0.585 \\
\hline WHO I & 80.0 & & 70.0 & & 79.7 & & 89.5 & & 78.3 & \\
\hline WHO II & 83.3 & & 75.0 & & 87.1 & & 91.6 & & 87.5 & \\
\hline WHO III & 80.9 & & 70.1 & & 91.4 & & 89.1 & & 77.4 & \\
\hline T stage & & 0.334 & & 0.329 & & 0.790 & & 0.103 & & 0.579 \\
\hline T1-2 & 87.4 & & 61.5 & & 90.4 & & 81.0 & & 72.6 & \\
\hline T3-4 & 79.7 & & 73.0 & & 88.6 & & 91.3 & & 80.2 & \\
\hline N stage & & 0.002 & & $<0.001$ & & 0.241 & & 0.009 & & $<0.001$ \\
\hline N0-1 & 94.8 & & 89.6 & & 93.0 & & 98.2 & & 98.3 & \\
\hline $\mathrm{N} 2-3$ & 74.8 & & 62.2 & & 87.1 & & 85.6 & & 70.3 & \\
\hline Overall stage & & $<0.001$ & & 0.002 & & 0.008 & & 0.115 & & $<0.001$ \\
\hline III & 90.2 & & 78.0 & & 93.3 & & 91.7 & & 87.3 & \\
\hline IVA and IVB & 63.2 & & 56.8 & & 80.4 & & 84.9 & & 62.3 & \\
\hline Regimen & & 0.575 & & 0.469 & & 0.211 & & 0.485 & & 0.369 \\
\hline NF group & 82.4 & & 72.6 & & 86.2 & & 91.0 & & 80.7 & \\
\hline PF group & 79.4 & & 68.7 & & 92.6 & & 87.7 & & 77.0 & \\
\hline NAC cycle & & 0.226 & & 0.230 & & 0.171 & & 0.472 & & 0.319 \\
\hline 1 & 89.5 & & 78.2 & & 96.3 & & 93.0 & & 84.9 & \\
\hline $2-3$ & 79.5 & & 69.4 & & 87.6 & & 88.8 & & 77.7 & \\
\hline
\end{tabular}

OS, overall survival; PFS, progression-free survival; LRFS, local relapse-free survival; RRFS, regional relapse-free survival; DMFS, distant metastasis-free survival; NAC, neoadjuvant chemotherapy.

\section{Prognostic factors}

To determine which factors affected patient outcome, we used univariate analyses to evaluate the prognostic value of gender, age, histology, $\mathrm{T}$ stage, $\mathrm{N}$ stage, overall stage, regimen group and cycle of neoadjuvant chemotherapy (Table 4). The outcomes showed that 5-year OS, PFS, RRFS and DMFS of patients with N0-1 stage were superior to those of N2-3 stage (OS: $94.8 \%$ vs $74.8 \%$, P $=0.002$; PFS: $89.6 \%$ vs $62.2 \%, \mathrm{P}<0.001$; RRFS: $98.2 \%$ vs $85.6 \%, \mathrm{P}=0.009$; DMFS: $98.3 \%$ vs $70.3 \%, \mathrm{P}<0.001$ ), and patients with clinical stage IV were inferior to those patients with stage III (OS: $63.2 \%$ vs $90.2 \%$, P<0.001; PFS: $56.8 \%$ vs $78.0 \%, \mathrm{P}=0.002$; LRFS: $80.4 \%$ vs $93.3 \%, \mathrm{P}=0.008$; DMFS: $62.3 \%$ vs $87.3 \%, \mathrm{P}<0.001)$. In multivariate analysis (Table 5), $\mathrm{N}$ stage was an independent factor for OS, PFS, RRFS and DMFS.

\section{Discussion}

Our research is a retrospective analysis for the treatment of locally advanced NPC with PF or NF regimen neoadjuvant chemotherapy combined with concurrent chemoradiotherapy. Compared with the PF group, the NF group showed a similar efficacy in the tumor response rate. As shown in table 2, there were no significant differences in $\mathrm{CR}$ rate both in the response of primary tumor and nodes between the two groups. In the present study, we also found that the PF group and the NF group had similar survival outcomes. The 5-year OS, PFS, LRFS, RRFS, and DMFS rates did not differ significantly between the two groups. In terms of acute toxics effects, the PF group suffered higher incidents of grade 3 to 4 nausea, vomiting and weight loss than the NF group, while the incidences of neutropenia and thrombocytopenia were lower in the PF group. Among the potential prognostic factors examined, we indicated that $\mathrm{N}$ stage were independent prognostic factors of outcome.

Table 5. Impact of prognostic factors on treatment results by multivariate analysis ( $P$ value)

\begin{tabular}{lccccc}
\hline Factors & 5y-OS & 5y-PFS & 5y-LRFS & 5y-RRFS & 5y-DMFS \\
\hline $\begin{array}{l}\text { Gender } \\
\text { Male vs. Female }\end{array}$ & 0.929 & 0.323 & 0.949 & 0.404 & 0.423 \\
$\begin{array}{l}\text { Age } \\
\leq 50 \text { vs. }>50\end{array}$ & 0.667 & 0.354 & 0.515 & 0.685 & 0.525 \\
$\begin{array}{l}\text { Regimen } \\
\text { NF vs. PF }\end{array}$ & 0.630 & 0.534 & 0.211 & 0.550 & 0.425 \\
$\begin{array}{l}\text { T stage } \\
\text { T1-2 vs. T3-4 }\end{array}$ & 0.077 & 0.832 & 0.790 & 0.467 & 0.496 \\
$\begin{array}{l}\text { N stage } \\
\text { N0-1 vs. N2-3 }\end{array}$ & $\mathbf{0 . 0 0 6}$ & $\mathbf{0 . 0 0 1}$ & 0.241 & $\mathbf{0 . 0 3 1}$ & $\mathbf{0 . 0 0 4}$
\end{tabular}

OS, overall survival; PFS, progression-free survival; LRFS, local relapse-free survival; RRFS, regional relapse-free survival; DMFS, distant metastasis-free survival.

Cisplatin-based NACT or CCRT combined with IMRT can achieve better clinical outcome than radiotherapy alone and has being the standard treatment for locally advanced NPC $[6,7,9,24]$. The main treatment failure pattern is distant metastasis [25]. Ferrari et al [26] reported that long-term outcome of locoregionally advanced nasopharyngeal carcinoma patients treated with cisplatin-based induction chemotherapy and current chemoradiotherapy. The 3-year overall survival and progression-free survival rates are $80.0 \%$ and $54.0 \%$, respectively. $\mathrm{Wu}$ et al [27] performed a prospective, multicenter clinical study to evaluate the long-term outcome in III-IVb NPC patients treated with IMRT with concurrent CDDP chemotherapy. The 5-year actuarial rates of OS, LRFS, RRFS, and DMFS were $78.4 \%, 86.8 \%, 88.4 \%$, and $78.0 \%$, respectively. Xu et al [28] demonstrated that the 3-year LRFS, DMSF, PFS and OS rates were $90.3 \%, 79.7 \%, 77.3 \%$, and $81.6 \%$ for the locoregionally advanced NPC patients treated with concurrent chemoradiotherapy with NDP plus fluorouracil. In our study, we observed the similar results. The 5-year OS, PFS, LRFS, RRFS and DMFS in the NF and PF groups were $82.4 \%$ vs $79.4 \%, 72.6 \%$ vs $68.7 \%, 86.2 \%$ vs $92.6 \%, 91.0 \%$ vs $87.7 \%, 80.7 \%$ vs $77.0 \%$, respectively. Furthermore, According to our results, $\mathrm{N}$ stage was an independent factor for OS, PFS, RRFS and DMFS, which was comparable to the results published by Lin et al [29] and Lai et al [30].

In terms of the non-hematological toxicities, the PF group resulted in a higher frequency of grade 3 to 
4 nausea and vomiting than the NF group ( $0 \%$ vs. $13.3 \%, P<0.001)$. According to the previous studies, the incidences of severe digestive toxicity was about $27.3 \%-32.1 \%$ in cisplatin-based chemoradiotherapy $[31,32]$. Our research revealed a much lower frequency of moderate to severe nausea and vomiting, it might due to the use of new generation of antiemetic drugs and better supportive care. Furthermore, we found that the incidence of grade 3-4 weight loss is higher in the PF group than in the NF group ( $4.8 \%$ vs $0 \%, P=0.025)$, which might be related with the gastrointestinal toxicities caused by CDDP. Additionally, another remarkable side effect of CDDP is renal toxicity. However, in our research, we did not observe grade 3-4 renal toxicity in both groups, which may due to the use of pre- and post-hydration and divide the dose of CDDP into 3 days. With respect to the hematological toxicities, the NF group resulted in a higher frequency of grade 3 to 4 neutropenia (46.6\% vs. $31.3 \%, \mathrm{P}=0.035)$ and thrombocytopenia $(17.5 \%$ vs. $7.3 \%, \mathrm{P}=0.042$ ) compared with the PF group, which is higher than those reported by Cao et al [20]. Lee et al [32] reported that the dose-limiting toxicity of NDP is bone marrow suppression, however, it was reversible and transient. Furthermore, thrombopoietin (TPO) and granulocyte-colony stimulating factor (G-CSF) were used when patients had bone marrow suppression. Thus, there were no treatment-related deaths in both groups in the current study.

In conclusion, our study demonstrated that neoadjuvant chemotherapy with 5-FU plus NDP combined with concomitant NDP and IMRT achieved comparable clinical outcome to those of cisplatin-based regiments, whereas the side effect is mild and acceptable. Therefore, neoadjuvant chemotherapy with 5-FU plus NDP combined with concomitant NDP and IMRT might be an effective and safe choice for patients with locally advanced NPC. As our research is a retrospective study with small sample, which may have many limitations, we are looking forward to have more well-designed, randomized, prospective, and large sample clinical research in the future.

\section{Acknowledgements}

This work was supported by the grants from the National Natural Science Foundation of China (NO. 81502647 and NO. 81502646) and the Zhejiang Medical and Health Science and Technology Project (NO. 2016146960 and NO. 2018260661).

\section{Competing Interests}

The authors have declared that no competing interest exists.

\section{References}

1. Cao SM, Simons MJ, Qian CN. The prevalence and prevention of nasopharyngeal carcinoma in China. Chin J Cancer 2011; 30: 114-119.

2. Chan AT. Nasopharyngeal carcinoma. Ann Oncol 2010; 21 Suppl 7: i308-i312.

3. Chan AT, Teo PM, Johnson PJ. Nasopharyngeal carcinoma. Ann Oncol 2002; 13: 1007-1015.

4. Wolden SL, Chen WC, Pfister DG, et al. Intensity-modulated radiation therapy (IMRT) for nasopharynx cancer: update of the Memorial Sloan-Kettering experience. Int J Radiat Oncol Biol Phys 2006; 64: 57-62.

5. Zhang L, Zhao C, Ghimire B, et al. The role of concurrent chemoradiotherapy in the treatment of locoregionally advanced nasopharyngeal carcinoma among endemic population: a meta-analysis of the phase III randomized trials. BMC Cancer 2010; 10: 558.

6. Chan AT, Teo PM, Ngan RK, et al. Concurrent chemotherapy-radiotherapy compared with radiotherapy alone in locoregionally advanced nasopharyngeal carcinoma: progression-free survival analysis of a phase III randomized trial. J Clin Oncol 2002; 20: 2038-2044.

7. Lin JC, Jan JS, Hsu CY, et al. Phase III study of concurrent chemoradiotherapy versus radiotherapy alone for advanced nasopharyngeal carcinoma: positive effect on overall and progression-free survival. J Clin Oncol 2003; 21: 631-637.

8. Lin JC, Liang WM, Jan JS, et al. Another way to estimate outcome of advanced nasopharyngeal carcinoma--is concurrent chemoradiotherapy adequate? Int J Radiat Oncol Biol Phys 2004; 60: 156-164.

9. Hui EP, Ma BB, Leung SF, et al. Randomized phase II trial of concurrent cisplatin-radiotherapy with or without neoadjuvant docetaxel and cisplatin in advanced nasopharyngeal carcinoma. J Clin Oncol 2009; 27: 242-249.

10. Fountzilas $\mathrm{G}$, Ciuleanu E, Bobos M, et al. Induction chemotherapy followed by concomitant radiotherapy and weekly cisplatin versus the same concomitant chemoradiotherapy in patients with nasopharyngeal carcinoma: a randomized phase II study conducted by the Hellenic Cooperative Oncology Group (HeCOG) with biomarker evaluation. Ann Oncol 2012; 23: 427-435.

11. Chen Y, Liu MZ, Liang SB, et al. Preliminary results of a prospective randomized trial comparing concurrent chemoradiotherapy plus adjuvant chemotherapy with radiotherapy alone in patients with locoregionally advanced nasopharyngeal carcinoma in endemic regions of china. Int J Radiat Oncol Biol Phys 2008; 71: 1356-1364.

12. Chen YP, Guo R, Liu N, et al. Efficacy of the Additional Neoadjuvant Chemotherapy to Concurrent Chemoradiotherapy for Patients with Locoregionally Advanced Nasopharyngeal Carcinoma: a Bayesian Network Meta-analysis of Randomized Controlled Trials. J Cancer 2015; 6: 883-892.

13. Tan T, Lim WT, Fong KW, et al. Concurrent chemo-radiation with or without induction gemcitabine, Carboplatin, and Paclitaxel: a randomized, phase 2/3 trial in locally advanced nasopharyngeal carcinoma. Int J Radiat Oncol Biol Phys 2015; 91: 952-960.

14. Sasaki Y, Tamura T, Eguchi K, et al. Pharmacokinetics of (glycolate-0,0')-diammine platinum (II), a new platinum derivative, in comparison with cisplatin and carboplatin. Cancer Chemother Pharmacol 1989; 23: 243-246.

15. Matsumoto $\mathrm{H}$, Hirabayashi $\mathrm{Y}, \mathrm{Kubota} \mathrm{H}$, et al. A combined therapy with docetaxel and nedaplatin for relapsed and metastatic esophageal carcinoma. Anticancer Res 2012; 32: 1827-1831.

16. Teramoto K, Asada Y, Ozaki Y, et al. A phase II study of docetaxel plus nedaplatin in patients with metastatic non-small-cell lung cancer. Cancer Chemother Pharmacol 2012; 70: 531-537.

17. Zhang MQ, Liu SP, Wang XE. Concurrent chemoradiotherapy with paclitaxel and nedaplatin followed by consolidation chemotherapy in locally advanced squamous cell carcinoma of the uterine cervix: preliminary results of a phase II study. Int J Radiat Oncol Biol Phys 2010; 78: 821-827.

18. Nakamura T, Kodaira T, Tachibana H, et al. Chemoradiotherapy for locally recurrent nasopharyngeal carcinoma: treatment outcome and prognostic factors. Jpn J Clin Oncol 2008; 38: 803-809.

19. Nakamura T, Kodaira T, Tachibana $\mathrm{H}$, et al. Clinical outcome of oropharyngeal carcinoma treated with platinum-based chemoradiotherapy. Oral Oncol 2009; 45: 830-834.

20. Cao KJ, Zhang AL, Ma WJ, et al. Nedaplatin or cisplatin combined with 5-fluorouracil for treatment of stage III-IVa nasopharyngeal carcinoma: a randomized controlled study. Zhonghua Zhong Liu Za Zhi 2011; 33: 50-52.

21. Tang LQ, Chen DP, Guo L, et al. Concurrent chemoradiotherapy with nedaplatin versus cisplatin in stage II-IVB nasopharyngeal carcinoma: an open-label, non-inferiority, randomised phase 3 trial. Lancet Oncol 2018; 19: 461-473.

22. Edge SB, Compton CC. The American Joint Committee on Cancer: the 7th edition of the AJCC cancer staging manual and the future of TNM. Ann Surg Oncol 2010; 17: 1471-1474.

23. Lee N, Harris J, Garden AS, et al. Intensity-modulated radiation therapy with or without chemotherapy for nasopharyngeal carcinoma: radiation therapy oncology group phase II trial 0225. J Clin Oncol 2009; 27: 3684-3690.

24. Kim K, Wu HG, Kim HJ, et al. Intensity-modulated radiation therapy with simultaneous integrated boost technique following neoadjuvant chemotherapy for locoregionally advanced nasopharyngeal carcinoma. Head Neck 2009; 31: 1121-1128.

25. Lee AW, Lin JC, Ng WT. Current management of nasopharyngeal cancer. Semin Radiat Oncol 2012; 22: 233-244. 
26. Wu F, Wang R, Lu H, et al. Concurrent chemoradiotherapy in locoregionally advanced nasopharyngeal carcinoma: treatment outcomes of a prospective, multicentric clinical study. Radiother Oncol 2014; 112: 106-111.

27. Ferrari D, Chiesa F, Codeca C, et al. Locoregionally advanced nasopharyngeal carcinoma: induction chemotherapy with cisplatin and 5-fluorouracil followed by radiotherapy and concurrent cisplatin: a phase II study. Oncology 2008; 74: 158-166.

28. $\mathrm{Xu} \mathrm{J}, \mathrm{He} \mathrm{X}, \mathrm{Cheng} \mathrm{K}$, et al. Concurrent chemoradiotherapy with nedaplatin plus paclitaxel or fluorouracil for locoregionally advanced nasopharyngeal carcinoma: Survival and toxicity. Head Neck 2014; 36: 1474-1480.

29. Lin S, Pan J, Han L, et al. Nasopharyngeal carcinoma treated with reduced-volume intensity-modulated radiation therapy: report on the 3-year outcome of a prospective series. Int J Radiat Oncol Biol Phys 2009; 75: 1071-1078.

30. Lai SZ, Li WF, Chen L, et al. How does intensity-modulated radiotherapy versus conventional two-dimensional radiotherapy influence the treatment results in nasopharyngeal carcinoma patients? Int J Radiat Oncol Biol Phys 2011; 80: 661-668.

31. Al-Sarraf M, LeBlanc M, Giri PG, et al. Chemoradiotherapy versus radiotherapy in patients with advanced nasopharyngeal cancer: phase III randomized Intergroup study 0099. J Clin Oncol 1998; 16: 1310-1317.

32. Lee AW, Tung SY, Chua DT, et al. Randomized trial of radiotherapy plus concurrent-adjuvant chemotherapy vs radiotherapy alone for regionally advanced nasopharyngeal carcinoma. J Natl Cancer Inst 2010; 102: 1188-1198. 